\title{
Risco de geada e duração dos subperíodos fenológicos da 'Cabernet Sauvignon' na região da Campanha ${ }^{1}$
}

\author{
Gabriel F. Brixner ${ }^{2}$, Edgar R. Schöffel ${ }^{3}$, Isabel Lago ${ }^{4}$, André L. Radünz ${ }^{5}$ \& Alexandra P. Krüger ${ }^{6}$ \\ ${ }^{1}$ Parte da Dissertação do primeiro autor, apresentada ao Programa de Pós-Graduação em Sistemas de Produção Agrícola Familiar da Universidade Federal de Pelotas \\ ${ }^{2}$ PPGA/UFSM. Santa Maria, RS. E-mail: brixner_gfb@yahoo.com.br (Autor correspondente) \\ ${ }^{3}$ FAEM/UFPel. Pelotas, RS. E-mail: ricardo_schoffel@ufpel.edu.br \\ ${ }^{4}$ UFSM. Santa Maria, RS. E-mail: isalago08@yahoo.com.br \\ ${ }^{5}$ PPGA/UFPel. Pelotas, RS. E-mail: alradunz@yahoo.com.br \\ ${ }^{6}$ UFPel. Pelotas, RS. E-mail: alexandra_kruger@hotmail.com
}

\section{Palavras-chave:}

fenologia

graus-dia (GD)

necessidade térmica

probabilidade

Vitis vinifera

\section{R E S U M O}

Objetivou-se, neste trabalho, identificar os períodos com maior risco de formação de geada e avaliar as alterações quanto à duração do ciclo fenológico da videira 'Cabernet Sauvignon', estimados com base na disponibilidade térmica de Alegrete, Bagé, Quaraí, São Gabriel, Santana do Livramento e Uruguaiana, localizados na região da Campanha do Rio Grande do Sul. Foram utilizados dados meteorológicos, como temperatura máxima e mínima do ar para o período de 1961 a 2010. Para determinar o risco de formação de geada foi considerada condição favorável a formação quando a temperatura mínima fosse menor ou igual a $3^{\circ} \mathrm{C}$. Para determinar o comportamento fenológico da cultivar Cabernet Sauvignon foi realizado o somatório de graus-dia abrangendo o período de 1/ agosto a 10/outubro como início de brotação. Verifica-se que, no início de setembro, Uruguaiana e São Gabriel apresentam baixo risco de formação de geada, a nível de 0,05, enquanto para Alegrete, Bagé, Quaraí e Santana do Livramento para este nível a última data ocorre no início de outubro. Quanto aos subperíodos de desenvolvimento da 'Cabernet Sauvignon' é possível observar variações no número total de dias em relação às épocas de brotação, bem como entre os municípios da Campanha.

\section{Key words:}

phenology

degrees-day (DD)

thermal requirement

probability

Vitis vinifera

\section{Risk of frost and duration of the phenological subperiods of 'Cabernet Sauvignon' in the Campanha}

\begin{abstract}
A B S T R A C T
The study aimed to identify the periods with higher risk of frost formation and to evaluate the changes regarding the duration of the phenological cycle of the vine Cabernet Sauvignon, based on the thermal availability of Alegrete, Bagé, Quaraí, São Gabriel, Santana do Livramento and Uruguaiana, located in the Campanha region of Rio Grande do Sul state. The meteorological data such as maximum and minimum air temperature for the period from 1961 to 2010 were used. In order to determine the risk of frost formation, it was considered as favorable condition for the formation when the minimum temperature was less than or equal to $3{ }^{\circ} \mathrm{C}$. To determine the phenological behavior of the cultivar Cabernet Sauvignon the sum of degrees-day, covering the period from August 1 to October 10 as the beginning of the sprouting. It was found that Uruguaiana and São Gabriel in early September had low frost formation risk, at $5 \%$ level, while in the other municipalities the last date occurs in early October. As for the development subperiod of Cabernet Sauvignon it is possible to observe variations in the total number of days in relation to the sprouting periods, as well as among the municipalities of Campanha.
\end{abstract}

\section{INTRODUÇÃO}

O Brasil ocupa a terceira posição em área plantada com vinhedos na América do Sul (Regina, 2006). O Rio Grande do Sul é o maior produtor brasileiro (Brunetto et al., 2007) com quatro regiões vitivinícolas: Serra Gaúcha, Campanha, Serra do Sudeste e Campos de Cima da Serra dentre as quais a região da Campanha se vem destacando nos últimos anos, na vitivinicultura gaúcha.

O setor da vitivinicultura da Campanha vem-se consagrando no cultivo de cultivares como: Chardonnay, Riesling Itálico,
Trebbiano, (brancas), Cabernet Franc, e Cabernet Sauvignon e Merlot (tintas). Sendo que 'Cabernet Sauvignon' é a vinífera tinta mais importante do Estado, é de brotação e maturação tardias, relativamente vigorosas, de média produção e elevada qualidade para vinificação (Rizzon \& Mielle, 2002).

A determinação do desenvolvimento da cultura frente às condições climáticas, como o risco de geada e a disponibilidade térmica, são parâmetros importantes na escolha das regiões com aptidão para a vitivinicultura uma vez que são variáveis que influenciam, direta e indiretamente, na produtividade e na qualidade final das uvas produzidas. 
Assim, o estudo do risco de ocorrência de geadas na região da Campanha torna-se necessário pois geadas primaveris podem causar a destruição dos órgãos herbáceos da planta (Barrera et al., 2007). Os sintomas de danos podem ser percebidos nos tecidos jovens e gemas em início de brotação, que se tornam flácidos, chamuscados e de coloração caramelada, secando posteriormente (Mandelli et al., 2009).

Um aspecto relevante a ser considerado nesses estudos, é que este é um fenômeno que ocorre na superfície terrestre. Assim, uma maneira bastante simples para a sua determinação é o uso da temperatura mínima da relva para expressar a intensidade do evento (Oliveira et al., 1997) e para medir a frequência de ocorrência em determinado período.

Em estudos similares, devido à maior disponibilidade de dados, tem sido utilizada a temperatura do ar medida em abrigo meteorológico em substituição à temperatura de relva. As variações de temperatura entre essas duas condições dependem do gradiente adiabático do momento, porém vários autores assumem que a temperatura do abrigo meteorológico supera em $4{ }^{\circ} \mathrm{C}$ a temperatura mínima na relva (Grodzki et al., 1996; Oliveira et al., 1997; Silva \& Sentelhas, 2001; Wrege et al., 2005; Caramori et al., 2008; Almeida et al., 2009). Desta forma, temperatura de $3^{\circ} \mathrm{C}$ no abrigo meteorológico equivale à temperatura em torno de $-1{ }^{\circ} \mathrm{C}$ na relva apresentando condições favoráveis à formação de geada e com possíveis danos às videiras.

Por outro lado, o conhecimento das datas de ocorrência dos eventos fenológicos por meio das necessidades térmicas, é uma técnica da viticultura moderna que auxilia na otimização do processo produtivo, de vez que possibilita a racionalização e o melhor planejamento das práticas culturais que são indispensáveis para o cultivo da videira (Mandelli et al., 2003; Tecchio et al., 2011). Sabe-se que o desenvolvimento vegetativo varia em função das características intrínsecas de cada cultivar, da época de poda e das condições meteorológicas de cada safra (Leão \& Silva, 2003; Brixner et al., 2010).

A relação entre fenologia e necessidades térmicas das videiras cultivadas no Rio Grande do Sul são abordadas por Schiedeck et al. (1997), Mandelli et al. (2003), Chavarria et al. (2009), Brixner et al. (2010), Costa (2011), Anzanello et al. (2012) e Radünz et al. (2012) os quais constatam que em anos ou locais com menor disponibilidade térmica as videiras demonstraram aumento na duração do ciclo.

Considerando a variabilidade da disponibilidade térmica nas condições climáticas do Sul do Brasil, são previstas alterações quanto à data da brotação e ao número de dias para completar o ciclo das videiras nessas condições, motivo pelo qual se tornam imprescindíveis estudos que contemplem uma série histórica de dados meteorológicos (normal climatológica), podendo-se quantificar, em dias, a fenologia das plantas para cada época de brotação com sua respectiva da data de ocorrência. Porém se necessita, para um estudo desse tipo, que a necessidade térmica utilizada como referência para estimar os eventos fenológicos seja, preferencialmente, representativa das condições climáticas da região de estudo e que tenha consistência significativa no número de anos analisado. Desta forma se destacam dois trabalhos, desenvolvidos nas condições da região da Campanha, Brixner et al. (2010) e Costa (2011).

'Cabernet Sauvignon' Brixner et al. (2010) observaram requerimento térmico de $2.208 \mathrm{GD}$ enquanto Costa (2011) observou requerimento térmico de $1.926 \mathrm{GD}$; esta diferença deve estar relacionada ao estádio fenológico em que se iniciou o acúmulo térmico sendo que Brixner et al. (2010) a consideraram a partir da poda e Costa (2011) a admitiu a partir do início da brotação.

Com isto, o presente trabalho tem, como objetivos, identificar os períodos com maior risco de formação de geada tal como avaliar as alterações quanto à duração do ciclo fenológico da videira 'Cabernet Sauvignon', estimados com base na disponibilidade térmica de Alegrete, Bagé, Quaraí, São Gabriel, Santana do Livramento e Uruguaiana, localizados na Campanha do Rio Grande do Sul.

\section{Material e Métodos}

O estudo teve como abrangência alguns municípios da região da Campanha do Rio Grande do Sul (RS), conforme descrito no macrozoneamento agroecológico e econômico do estado do Rio Grande do Sul (Maluf \& Westphalen, 1994). Esta região é composta por 14 municípios e dividida em duas subregiões: Fronteira Uruguai e Uruguaiana/São Gabriel (Figura 1).

Dados diários de temperaturas máxima e mínima do ar foram realizados para o período de 1961 a 2010, de seis postos meteorológicos, sendo eles: Bagé, Quaraí e Santana do Livramento (sub-região Fronteira Uruguai) e Alegrete, São Gabriel, Uruguaiana (sub-região Uruguaiana/São Gabriel) com uma série mínima de observações diárias de 25 anos. Referidos

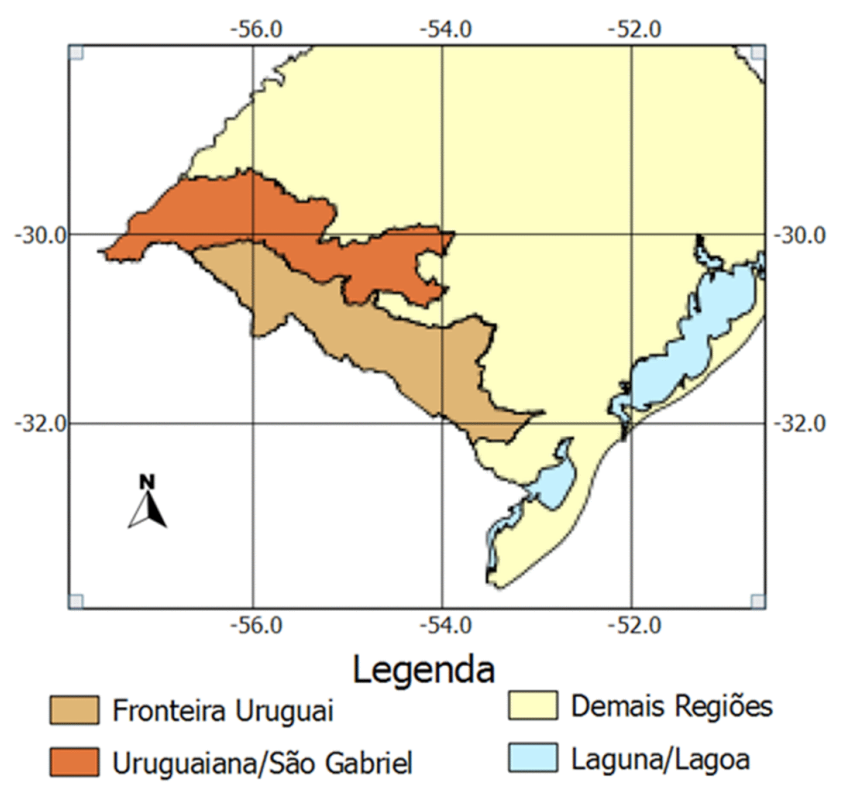

Figura 1. Região da Campanha do Rio Grande do Sul (RS) dividida em duas sub-regiões: Fronteira Uruguai e Uruguaiana/São Gabriel, conforme descrito no macrozoneamento agroecológico e econômico do estado do Rio Grande do Sul 
dados foram organizados em planilhas eletrônicas formando um banco de dados estruturado de forma sequencial; esses dados foram obtidos junto ao $8^{\circ}$ DISME/INMET e à FEPAGRO.

Para determinação do risco de geada considerou-se o período entre $01 /$ abril a 12 /novembro, por serem as datas limites mais próximas ao primeiro e ao último evento durante a série analisada, respectivamente. A frequência diária em que a temperatura mínima apresentou valores iguais ou inferiores a $3{ }^{\circ} \mathrm{C}$, correspondeu como condição favorável à formação de geada. Desta forma se registrou, quando a condição foi satisfeita, o valor "1", caso contrário "0", e a média do número de vezes desta condição estabeleceu a probabilidade multiplicando-se a média por 100 para transformá-la em porcentagem (\%).

Para o requerimento térmico da 'Cabernet Sauvignon' foi utilizado o trabalho de Costa (2011) o qual estabeleceu a necessidade de 106,6 GD, do Início ao Fim da Brotação (IB-FB); de 231,6 GD, do Fim da Brotação ao Início da Floração (FB-IF); de 135,8 GD, do Início ao Fim da Floração (IF-FF); 725,1 GD, do Fim da Floração ao Início da Maturação (FF-IM) e 727,8 GD, do Início ao Fim da Maturação (IM-FM).

As datas dos estádios fenológicos foram simuladas para o período 01/agosto a 10/outubro, correspondendo ao início da brotação e determinadas por meio da disponibilidade térmica para toda a série histórica estudada (1961-2010), conforme disponibilidade de dados para cada município, pelo método de Graus-Dia (GD) (Tecchio et al., 2011; Anzanello et al., 2012), conforme Eq. (1):

$$
\mathrm{GD}=\sum_{\mathrm{i}=1}^{\mathrm{n}}\left(\frac{\mathrm{T}_{\mathrm{mx}}+\mathrm{T}_{\mathrm{mn}}}{2}\right)-\mathrm{T}_{\mathrm{b}}
$$

sendo:

GD - graus-dia $\left({ }^{\circ} \mathrm{C}\right)$, somatório do início da brotação $(\mathrm{i}=$ 1) até estádio fenológico (n)

$\mathrm{T}_{\mathrm{mx}}$ - temperatura máxima do ar, ${ }^{\circ} \mathrm{C}$

$\mathrm{T}_{\mathrm{mn}}$ - temperatura mínima do ar, ${ }^{\circ} \mathrm{C}$

$\mathrm{T}_{\mathrm{b}}$ - temperatura base, ${ }^{\circ} \mathrm{C}$, equivalente a $10{ }^{\circ} \mathrm{C}$ (Pedro Júnior et al., 1994b)

A fim de definir a data de ocorrência das somas térmicas para atingir os diferentes subperíodos fenológicos e calcular o número de dias dos períodos, foi desenvolvido um programa em VBA (Visual Basic for Applications) utilizado em planilha eletrônica. O número médio de dias de cada subperíodo fenológico e sua provável data de ocorrência são apresentados em intervalos de três dias, os quais foram obtidos por meio dos valores médios deste intervalo. Os valores apresentados para a região da Campanha são oriundos das médias dos resultados obtidos para os seis postos meteorológicos localizados nesta região.

\section{Resultados e Discussão}

A frequência de ocorrência de geada permitiu identificar um período em comum para toda a região da Campanha, entre o final do mês de maio e o final do mês de agosto, correspondendo ao período mais suscetível à formação de geada como pode ser observado pela Figura 2 que, neste período, não apresenta frequência igual a 0 o que representa aproximadamente 100 dias que a temperatura mínima absoluta do ar atinge pelo menos $3^{\circ} \mathrm{C}$. Resultado semelhante foi observado por Almeida

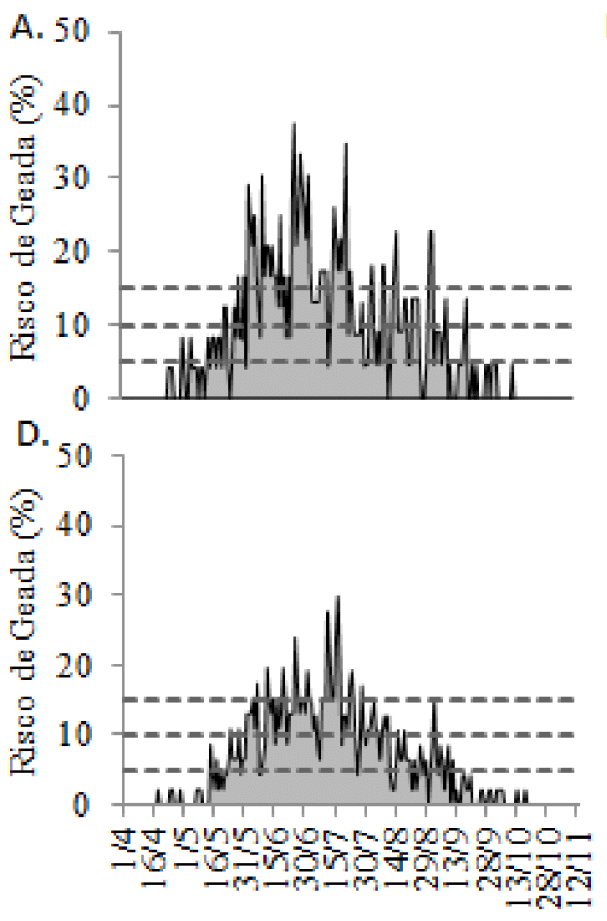

B.

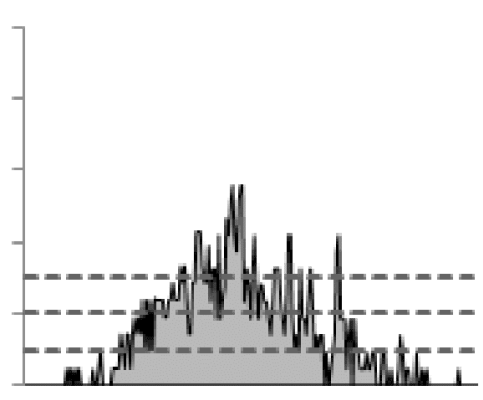

E.

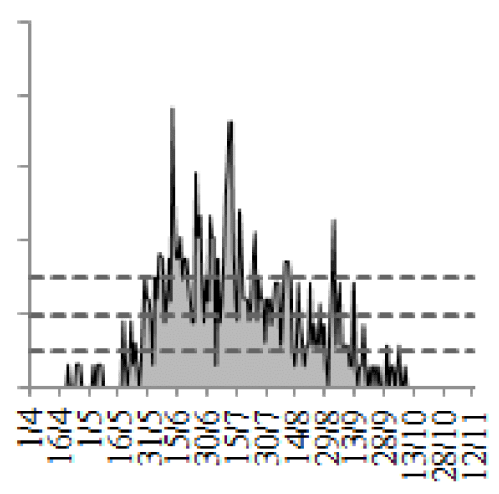

C.

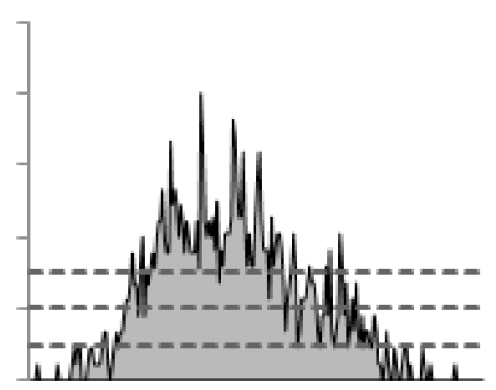

$\mathrm{F}$.

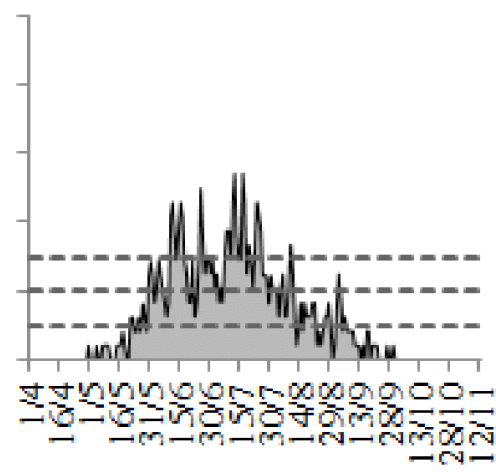

Figura 2. Risco diário de formação de geada com destaque para os níveis de 5, 10 e 15\%, para o período de $1^{\circ}$ de abril a 12 de novembro, para Alegrete (A.), Bagé (B.), Quaraí (C.), São Gabiel (D.), Sanatana do Livramento (E.) e Uruguaiana (F.), localizados na região da Campanha do Rio Grande do Sul, Brasil, no período de 1961 a 2010 
et al. (2009), no zoneamento agroclimático do morango no Rio Grande do Sul, que obtiveram, ao considerar a mesma temperatura de $3{ }^{\circ} \mathrm{C}$ medida em abrigo meteorológico, como crítico, o mesmo período do ano para 35 postos meteorológicos distribuídos por todo o Estado.

Nos seis municípios estudados o maior risco de geada em um mesmo dia foi de 40\% em Quaraí (Figura 2C) e os meses de junho e julho como aqueles com condições mais favoráveis à formação de geada. Na região do Baixo Vale do Taquari, RS, Buriol et al. (2000) também encontraram a maior frequência diária de ocorrência de temperaturas mínimas nesses meses.

Apesar do alto risco de geada que ocorre em junho, esta é uma época do ano em que ainda não ocorreu brotação das videiras; consequentemente, não haverá danos físicos às plantas. Além disto, geadas nesta época podem proporcionar a eliminação de alguns parasitas, como sugerem Mandelli et al. (2009).

Na Serra Gaúcha as videiras começam a brotar no final do inverno e no início da primavera dependendo da cultivar: as precoces iniciam a brotação até o dia 10 de setembro; as de ciclo médio a iniciam entre os dias 11 e 20 de setembro e as de ciclo tardio se iniciam após o dia 20 de setembro (Mandelli et al., 2003). Comportamento semelhante foi observado por Costa (2011), para quatro viníferas em Santana do Livramento.

No mês de agosto, sobretudo no terceiro decêndio, existe frequência em torno de $10 \%$ de ocorrência de geada equivalendo, em média, a uma geada a cada 10 anos (Figura 2). A última data com risco de ocorrência de geada superior a $10 \%$ foi próximo ao dia 2 de setembro, em São Gabriel, Uruguaiana e Bagé, e nos dias 10, 12 e 20 de setembro para Quaraí, Santana do Livramento e Alegrete, respectivamente. Na Figura 2 é possível visualizar que para o dia 2 de setembro, com exceção de São Gabriel e Uruguaiana, os demais municípios apresentaram frequência próxima a $20 \%$ ou superior, como Alegrete e Santana do Livramento com $23 \%$.

Verifica-se que na Campanha o primeiro decêndio de setembro tem elevada frequência de formação de geadas, o que foi observado também por Maluf et al. (2011) ao destacarem que apesar da região não apresentar altitude elevada apresenta frequência significativa de formação de geada para o primeiro decêndio de setembro, fato este influenciado pela permanência, neste mês, de entrada de massas de ar polar no Estado, a partir da região da Campanha.

Para os municípios de São Gabriel e Uruguaiana a última data com formação de geada a nível de $15 \%$ no período analisado, ocorre no primeiro decêndio de agosto (Figura 2D e F) enquanto para os demais municípios ocorre no primeiro decêndio de setembro. Destaca-se que mesmo em setembro o valor do risco atinge 14,8\% em São Gabriel (Figura 2D) e isto representa aproximadamente condição favorável à formação de uma geada a cada 7 anos.

Segundo Barrera et al. (2007) as brotações e os cachos podem ser danificados com temperaturas inferiores a $-0,5^{\circ} \mathrm{C}$. $\mathrm{Na}$ Campanha após a última data de formação de geada com risco de 10 e $5 \%$, as temperaturas mínimas absolutas foram - 0,4 e $-0,2{ }^{\circ} \mathrm{C}$, respectivamente, em São Gabriel; para o limite de $5 \%$ com exceção de São Gabriel, todas as regiões apresentaram valores superiores a $0{ }^{\circ} \mathrm{C}$.

Desta forma, brotações que ocorrem em época que apresenta $5 \%$ de risco de geada apresentam baixa probabilidade de sofrer danos físicos provocados pelas baixas temperaturas. Esta condição é obtida a partir de 5 e 11 de setembro em Uruguaiana e São Gabriel, respectivamente, e a partir de outubro em Santana do Livramento (dia 5), Bagé (dia 12), Alegrete e Quaraí (dia 13) (Figura 2).

Oliveira et al. (1997) analisaram a ocorrência de geada no Rio Grande do Sul através de estudo com dados decendiais de temperatura mínima absoluta do ar, com base no período de 1944 a 1993. Os autores verificaram que a região da Campanha apresenta elevado risco de formação de geada, do primeiro decêndio de agosto ao segundo de outubro, variando de 64 a 16\%.

Nota-se que esses autores encontraram probabilidade maior que a obtida no presente trabalho; esta diferença pode ser atribuída à maneira como foi calculada a probabilidade nesses trabalhos haja vista que Oliveira et al. (1997) determinaram a probabilidade com base na temperatura mínima absoluta do ar por decêndio enquanto neste foi em função da temperatura mínima diária diminuindo a frequência de temperaturas favoráveis à formação de geada.

A duração dos subperíodos fenológicos para a 'Cabernet Sauvignon' indica diminuição do número de dias, quanto mais tardia for a brotação (Tabela 1). Estudos similares para a videira 'Niagara Rosada', no estado de São Paulo, também constaram a mesma tendência (Pedro Júnior et al., 1994a; Bardin et al., 2010). Isto ocorre devido ao aumento gradativo da temperatura e, por consequência, da disponibilidade térmica do inverno para a primavera (Tabela 2).

A temperatura é considerada o elemento meteorológico de maior importância no desenvolvimento da videira controlando o ritmo dos vários estádios fenológicos, estando a possibilidade de cultivo da videira estreitamente ligada à fenologia da planta (Teixeira et al., 2002).

Baixas temperaturas nos estádios iniciais da videira causam, embora não formadoras de geadas, a paralisação do crescimento, o crestamento das folhas terminais dos ramos e o encurtamento dos meritalos (Mandelli et al., 2009). Quanto às temperaturas baixas no crescimento, essas peculiaridades merecem atenção nos meses iniciais (agosto e setembro) e nas regiões com menor disponibilidade térmica (Bagé, Quaraí e Santana do Livramento) (Tabela 2).

Brotações tardias se tornam uma alternativa para fugir de temperaturas não favoráveis pois ocorre redução no número de dias do ciclo da cultura quanto mais tardio for o início da brotação (Tabela 1). Por exemplo, comparando as brotações que se iniciam em 1 de agosto e 1 de setembro, a diferença é de 10 dias para a data provável de colheita (Tabela 1) porém a brotação em setembro fica menos suscetível a condições adversas, fato que pode interferir na produção. 
Tabela 1. Número médio de dias e desvio padrão da duração dos subperíodos fenológicos e data simulada de ocorrência dos principais estádios, para a videira 'Cabernet Sauvignon', no período de agosto ao início de outubro, na região da Campanha do Rio Grande do Sul, Brasil

\begin{tabular}{|c|c|c|c|c|c|c|c|c|c|c|}
\hline \multirow{3}{*}{$\begin{array}{l}\text { Data do } \\
\text { início da } \\
\text { brotação }\end{array}$} & \multicolumn{10}{|c|}{ Subperíodos fenológicos* } \\
\hline & \multicolumn{2}{|c|}{ IB - FB } & \multicolumn{2}{|c|}{ IB - IF } & \multicolumn{2}{|c|}{ IB - FF } & \multicolumn{2}{|c|}{ IB - IM } & \multicolumn{2}{|c|}{ IB $-F M$} \\
\hline & $\mathrm{N}^{0}$ dias & Data & $\mathrm{N}^{0}$ dias & Data & $\mathrm{N}^{0}$ dias & Data & $\mathrm{N}^{0}$ dias & Data & $\mathrm{N}^{0}$ dias & Data \\
\hline \multicolumn{11}{|l|}{ Agosto } \\
\hline 1 & $22 \pm 6$ & $24 / 08$ & $59 \pm 7$ & $30 / 09$ & $76 \pm 7$ & $16 / 10$ & $139 \pm 7$ & $19 / 12$ & $188 \pm 8$ & $06 / 02$ \\
\hline 4 & $22 \pm 5$ & $27 / 08$ & $58 \pm 7$ & $02 / 10$ & $75 \pm 7$ & $18 / 10$ & $137 \pm 7$ & $20 / 12$ & $186 \pm 8$ & $07 / 02$ \\
\hline 7 & $22 \pm 5$ & $29 / 08$ & $57 \pm 7$ & $04 / 10$ & $73 \pm 7$ & $20 / 10$ & $135 \pm 7$ & $21 / 12$ & $184 \pm 8$ & $08 / 02$ \\
\hline 10 & $21 \pm 5$ & $31 / 08$ & $56 \pm 7$ & $05 / 10$ & $71 \pm 6$ & $21 / 10$ & $133 \pm 7$ & $21 / 12$ & $182 \pm 8$ & $09 / 02$ \\
\hline 13 & $20 \pm 6$ & $03 / 09$ & $54 \pm 7$ & $07 / 10$ & $70 \pm 6$ & $22 / 10$ & $131 \pm 7$ & $22 / 12$ & $180 \pm 8$ & $10 / 02$ \\
\hline 16 & $20 \pm 6$ & $05 / 09$ & $53 \pm 6$ & $08 / 10$ & $68 \pm 6$ & $24 / 10$ & $129 \pm 7$ & $23 / 12$ & $178 \pm 8$ & $11 / 02$ \\
\hline 19 & $19 \pm 6$ & $08 / 09$ & $52 \pm 6$ & $10 / 10$ & $67 \pm 6$ & $25 / 10$ & $127 \pm 7$ & $24 / 12$ & $176 \pm 8$ & $12 / 02$ \\
\hline 22 & $19 \pm 6$ & $10 / 09$ & $50 \pm 6$ & $12 / 10$ & $65 \pm 6$ & $27 / 10$ & $125 \pm 7$ & $25 / 12$ & $174 \pm 8$ & $13 / 02$ \\
\hline 25 & $19 \pm 6$ & $13 / 09$ & $49 \pm 6$ & $14 / 10$ & $64 \pm 6$ & $28 / 10$ & $123 \pm 6$ & $27 / 12$ & $172 \pm 8$ & $14 / 02$ \\
\hline 28 & $18 \pm 5$ & $16 / 09$ & $48 \pm 5$ & $16 / 10$ & $62 \pm 5$ & $30 / 10$ & $121 \pm 6$ & $28 / 12$ & $170 \pm 8$ & $15 / 02$ \\
\hline \multicolumn{11}{|l|}{ Setembro } \\
\hline 1 & $18 \pm 5$ & $19 / 09$ & $47 \pm 5$ & $18 / 10$ & $61 \pm 5$ & $01 / 11$ & $119 \pm 6$ & $29 / 12$ & $168 \pm 8$ & $16 / 02$ \\
\hline 4 & $17 \pm 4$ & $21 / 09$ & $46 \pm 5$ & $20 / 10$ & $59 \pm 5$ & $03 / 11$ & $117 \pm 6$ & $30 / 12$ & $166 \pm 8$ & $18 / 02$ \\
\hline 7 & $16 \pm 4$ & $24 / 09$ & $44 \pm 5$ & $22 / 10$ & $58 \pm 5$ & $05 / 11$ & $115 \pm 6$ & $31 / 12$ & $164 \pm 8$ & $19 / 02$ \\
\hline 10 & $16 \pm 4$ & $26 / 09$ & $43 \pm 4$ & $24 / 10$ & $57 \pm 5$ & $06 / 11$ & $113 \pm 6$ & $02 / 01$ & $163 \pm 8$ & $20 / 02$ \\
\hline 13 & $15 \pm 3$ & $29 / 09$ & $42 \pm 4$ & $26 / 10$ & $55 \pm 5$ & $08 / 11$ & $111 \pm 6$ & $03 / 01$ & $161 \pm 8$ & $22 / 02$ \\
\hline 16 & $15 \pm 3$ & $01 / 10$ & $41 \pm 4$ & $27 / 10$ & $54 \pm 5$ & $10 / 11$ & $110 \pm 6$ & $04 / 01$ & $159 \pm 8$ & $23 / 02$ \\
\hline 19 & $14 \pm 3$ & $04 / 10$ & $40 \pm 4$ & $29 / 10$ & $53 \pm 5$ & $11 / 11$ & $108 \pm 6$ & $05 / 01$ & $158 \pm 8$ & $24 / 02$ \\
\hline 22 & $14 \pm 3$ & $06 / 10$ & $39 \pm 4$ & $31 / 10$ & $52 \pm 4$ & $13 / 11$ & $106 \pm 6$ & $07 / 01$ & $156 \pm 8$ & $26 / 02$ \\
\hline 25 & $13 \pm 3$ & $09 / 10$ & $38 \pm 4$ & $02 / 11$ & $51 \pm 4$ & $15 / 11$ & $105 \pm 6$ & $08 / 01$ & $155 \pm 8$ & $27 / 02$ \\
\hline 28 & $13 \pm 3$ & $11 / 10$ & $37 \pm 4$ & $05 / 11$ & $50 \pm 4$ & $17 / 11$ & $103 \pm 5$ & $10 / 01$ & $153 \pm 8$ & $01 / 03$ \\
\hline \multicolumn{11}{|l|}{ Outubro } \\
\hline 1 & $12 \pm 3$ & $14 / 10$ & $36 \pm 4$ & $07 / 11$ & $49 \pm 4$ & $19 / 11$ & $102 \pm 5$ & $11 / 01$ & $152 \pm 8$ & $02 / 03$ \\
\hline 4 & $12 \pm 2$ & $17 / 10$ & $35 \pm 3$ & $09 / 11$ & $48 \pm 4$ & $21 / 11$ & $100 \pm 5$ & $13 / 01$ & $151 \pm 8$ & $04 / 03$ \\
\hline 7 & $12 \pm 2$ & $20 / 10$ & $35 \pm 3$ & $12 / 11$ & $47 \pm 4$ & $23 / 11$ & $99 \pm 5$ & $15 / 01$ & $149 \pm 7$ & $06 / 03$ \\
\hline
\end{tabular}

*IB-FB -Início da Brotação ao Fim da Brotação; IB-IF -Início da Brotação ao Início da Floração; IB-FF - Início da Brotação a Fim da Floração; IB-IM - Início da Brotação a Início da Maturação; IB-FM - Início da Brotação ao Fim da Maturação

Tabela 2. Temperaturas médias decendiais para os meses de crescimento vegetativo em seis municípios da Campanha, RS, com base no período de 1961 a 2010

\begin{tabular}{|c|c|c|c|c|c|c|c|c|c|c|c|c|c|c|c|c|c|c|c|c|c|c|c|}
\hline \multirow{2}{*}{ Localidade } & \multicolumn{2}{|c|}{ Agosto } & \multicolumn{3}{|c|}{ Setembro } & \multicolumn{3}{|c|}{ Outubro } & \multicolumn{3}{|c|}{ Novembro } & \multicolumn{3}{|c|}{ Dezembro } & \multicolumn{3}{|c|}{ Janeiro } & \multicolumn{3}{|c|}{ Fevereiro } & \multicolumn{3}{|c|}{ Março } \\
\hline & $1^{*}$ & 2 & 1 & 2 & 3 & 1 & 2 & 3 & 1 & 2 & 3 & 1 & 2 & 3 & 1 & 2 & 3 & 1 & 2 & 3 & 1 & 2 & 3 \\
\hline Bagé & 13,0 & $13,714,7$ & 14,6 & 15,0 & 16,0 & 16,7 & 17,4 & 18,7 & 18,9 & 19,4 & 21,1 & 21,4 & 22,5 & 23,2 & 23,4 & 23,4 & 23,8 & 23,4 & 23,0 & 22,9 & 22,8 & 21,7 & 20,6 \\
\hline São Gabriel & 14,1 & $14,915,4$ & 15,7 & 16,1 & 17,1 & 18,0 & 18,7 & 20,0 & 20,4 & 20,9 & 22,2 & 22,7 & 23,8 & 24,2 & 24,6 & 24,8 & 24,9 & 24,4 & 23,9 & 24,1 & 23,8 & 22,7 & 21,8 \\
\hline $\begin{array}{l}\text { Santana do } \\
\text { Livramento }\end{array}$ & 12,6 & $12,913,6$ & 14,0 & 14,2 & 15,8 & 16,4 & 10,3 & 18,3 & 18,5 & 10 & 20,9 & 20,9 & & 22,5 & 23,0 & & 23,4 & 23 , & LC, & 22,3 & 22 , & 21 , & 20,3 \\
\hline
\end{tabular}

*1, 2 e 3 = decêndio

O subperíodo do IB-IM variou de acordo com a época de brotação, de 99 dias a 139 dias (Tabela 1). Nos demais subperíodos fenológicos a variação foi, em dias, menos expressiva. Nota-se, na Tabela 1, que os maiores desvios padrões em dias dos subperíodos fenológicos ocorreram com brotação no mês de agosto enquanto que os menores desvios tenderam a acontecer com as brotações que se iniciam em outubro.

De acordo com Costa (2011) na safra 1994/95 a 'Cabernet Sauvignon' apresentou somatório térmico de $1.926 \mathrm{GD}$, tendo as seguintes datas dos estádios: início de brotação no dia 6 de setembro, finalizando em 10 de outubro, iniciando-se a floração no dia 17 de outubro, finalizando em 7 de novembro, entrando em maturação no dia 22 de dezembro e finalizando em 15 de fevereiro. Analisando as datas dos subperíodos com o início da brotação em 4/setembro (período representativo do dia 6 de setembro) as datas simuladas estão abrangidas pelo desvio padrão (Tabela 1). A única exceção se refere ao subperíodo IB$\mathrm{FB}$, que apresentou divergência de aproximadamente 20 dias, se comparado ao obtido no trabalho de Costa (2011).

Além disto, as datas de início de brotação apresentadas por Costa (2011) variaram entre 18/agosto e 30/setembro, tendo data média $8 /$ setembro. Dentre as safras estudadas pelo autor somente a safra de 1993/94 se iniciou nesses dias apresentando coerência em relação às datas dos subperíodos fenológicos com 
os resultados obtidos na Tabela 1, novamente com exceção do IB-FB.

Esta divergência encontrada para o subperíodo IB-FB, tanto analisando a disponibilidade térmica como pela data de início de brotação, pode estar relacionada ao coeficiente de variação obtido neste subperíodo, por Costa (2011) ficando em torno de $60 \%$ porém, se verifica, apesar disto, consistência para estimar a data de ocorrência dos subperíodos fenológicos com base nos valores apresentados na Tabela 1.

Ressalta-se que o número total de dias e as prováveis datas de ocorrência dos subperíodos da 'Cabernet Sauvignon' (Tabela 1) correspondem aos valores médios dos seis municípios da Campanha, RS; observam-se, no entanto, diferenças de disponibilidade térmica entre os municípios tendo as maiores disponibilidades em Uruguaiana, Alegrete, São Gabriel e Quaraí e as menores em Santana do Livramento e Bagé (Tabela 2 e Figura 3). Neste sentido, no zoneamento climático
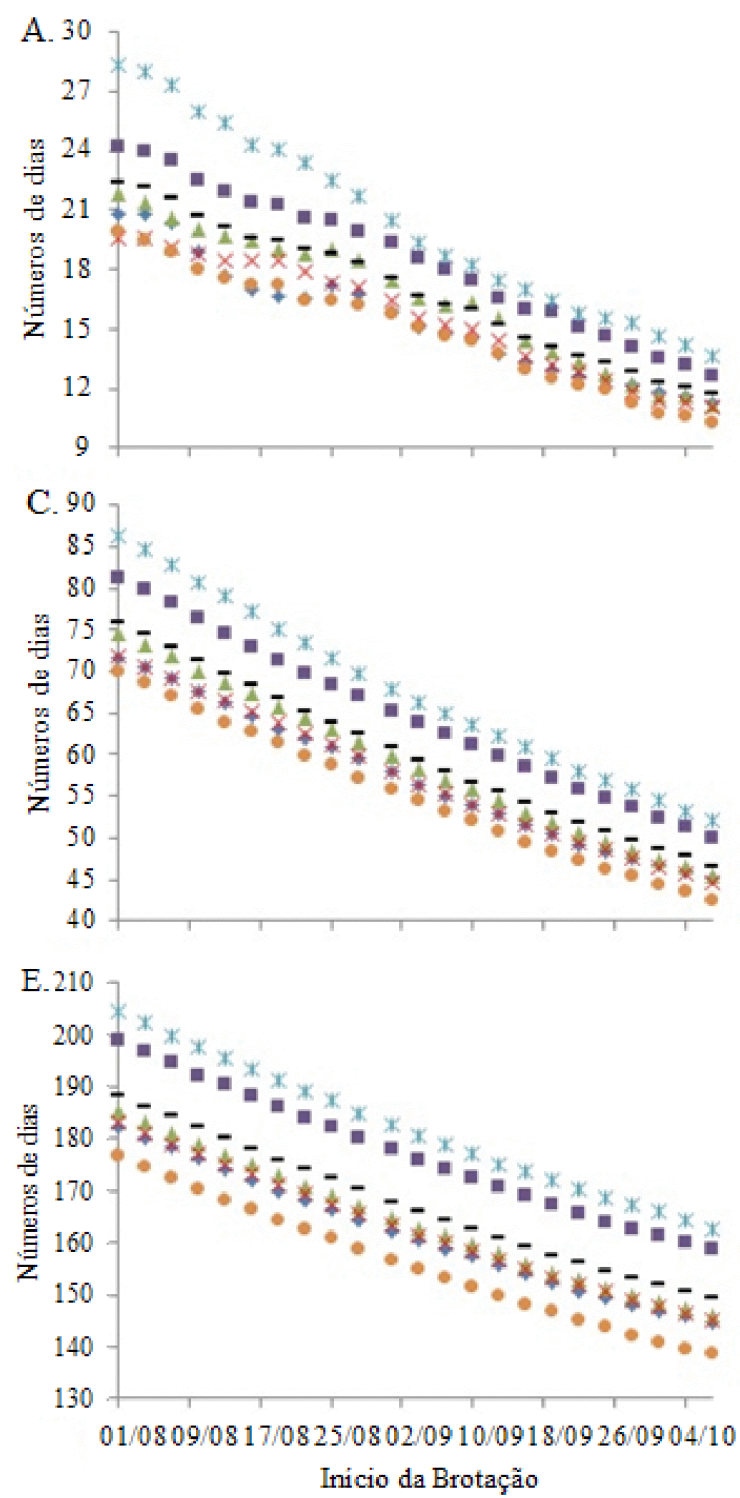

vitícola realizado para o Rio Grande do Sul por meio do Índice Heliotérmico, Tonietto et al. (2006) verificaram esta diferença permitindo distinguir esta região em duas classes térmicas, ambas com disponibilidade térmica para o cultivo de videiras incluindo cultivares de ciclo tardio.

Entre os municípios da região da Campanha, Santana do Livramento apresenta a menor disponibilidade térmica (Tabela 2 e Figura 3) necessitando de mais dias para completar a exigência da cultivar enquanto, por outro lado, as maiores disponibilidades ocorrem em Uruguaiana e São Gabriel atendendo, desta forma, à exigência térmica antes.

Nos municípios com maior disponibilidade as plantas irão apresentar brotação mais precoce porém principalmente no início do mês de agosto, esses dois municípios ainda apresentam risco significativo de formação de geada (Figura 2), destacandose São Gabriel que apresenta risco de $10 \%$ no primeiro decêndio de setembro (Figura 2D).
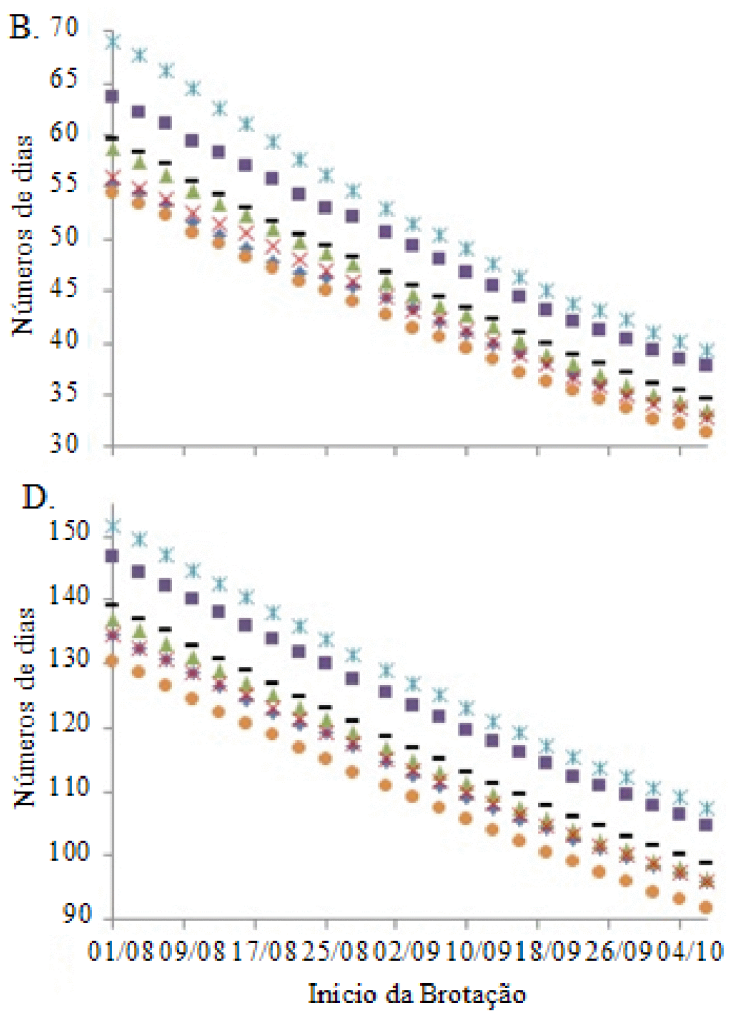

$\begin{array}{ll}\text { * Alegrete } & \text { * Santana do Livramento } \\ \text { - Bagé } & \text { - Unuguaiana } \\ \text { - Quarai } & \text { - Campanha } \\ \text { × São Gabriel } & \end{array}$

Figura 3.Duração em dias do início da brotação até o fim da brotação (A), início da floração (B), fim da floração (C), início da maturação (D) e fim da maturação (E), da videira 'Cabernet Sauvignon', com diferentes épocas de brotação para municípios da região da Campanha do Rio Grande do Sul, Brasil, no período de 1961 a 2010 
Nos demais municípios (Alegrete e Quaraí) em função de apresentarem menor disponibilidade térmica, as brotações podem ocorrer mais tarde mas se constata que ocorre redução na oscilação da temperatura; assim, as plantas estão menos suscetíveis a danos físicos causados pelas temperaturas baixas, em especial geadas (Figura 2A e C).

Em avaliação da fenologia das cvs. Cabernet Sauvignon, Merlot e Riesling Itálico em Uruguaiana e Quaraí, Amaral et al. (2009) verificaram que a brotação ocorreu antes em Uruguaiana sendo que a 'Riesling Itálico' nesta localidade sofreu influência por geadas mais severas, tendo como data de brotação 11/08.

$\mathrm{Na}$ Campanha os valores extremos do número total de dias foram, para todos os subperíodos, em Santana do Livramento e Uruguaiana (Figura 3). Além disto, dentre os subperíodos determinados o IB-FB apresentou maior variação entre os locais analisados sendo que neste subperíodo Alegrete tem maior variação no número de dias ao longo das épocas de brotação, seguido de São Gabriel (Figura 3A).

\section{Conclusões}

1. Os municípios de Uruguaiana e São Gabriel apresentam a última data com risco de formação de geada a nível de $15 \%$ no início de agosto, enquanto este mesmo risco se prolonga até o início de setembro em Santana do Livramento, Bagé, Alegrete e Quaraí.

2. Para o risco de formação de geada a nível de $5 \%$, São Gabriel e Uruguaiana apresentam a última data no início de setembro e Alegrete, Bagé, Quaraí e Santana do Livramento, no início de outubro, caracterizando-se como geadas primaveris.

3. A região da Campanha apresenta disponibilidade térmica para o cultivo da 'Cabernet Sauvignon' tendo, como período de início da brotação, primeiro de agosto a dez de outubro, cuja duração em dias dos subperíodos fenológicos varia em função da época em que ocorre a brotação e entre os municípios dessa região.

4. A cv. Cabernet Sauvignon apresenta ciclo vegetativo mais longo em dias em Santana do Livramento e Bagé do que em localidades mais quentes, como Uruguaiana, Alegrete, São Gabriel e Quaraí.

\section{Agradecimentos}

À Fundação Estadual de Pesquisa Agropecuária Gaúcha (FEPAGRO) e ao Instituto Nacional de Meteorologia - $8^{\circ}$ Distrito de Meteorologia (INMET - $8^{\circ}$ DISME) pela disponibilidade dos dados meteorológicos, fundamentais para a realização deste trabalho.

\section{Literatura Citada}

Almeida, I. R; Steinmetz, S.; Reisser Júnior, C; Antunes, L. E. C.; Alba, J. M. F.; Matzennauer, R.; Radin, B. Zoneamento agroclimático para produção de morango no Rio Grande do Sul. Pelotas: Embrapa Clima Temperado, 2009. 28p. Documentos, 283.
Amaral, U. do; Martins, C. R.; Filho, R. C.; Brixner, F. G.; Bini, D. A. Caracterização fenológica e produtiva de videiras Vitis vinifera L. cultivadas em Uruguaiana e Quaraí/RS. Revista da FZVA, v.16, p.22-31, 2009.

Anzanello, R.; de Souza, P. V. D.; Coelho, P. F. Fenologia, exigência térmica e produtividade de videiras 'Niagara Branca, 'Niagara Rosada' e 'Concord' submetidas a duas safras por ciclo vegetativo. Revista Brasileira de Fruticultura, v.34, p.366-376, 2012.

Bardin, L.; Pedro Júnior, M. J.; de Moraes, J. F. L.; Giardini, F. P. Estimativas das épocas de colheita da videira 'Niagara Rosada' na região do polo turístico do circuito das frutas, São Paulo. Scientia Agraria, v.11, p.135-139, 2010.

Barrera, L. M.; Gonzáles, A. I.; Parra, L. R. Efectos de las heladas en la agricultura. La Serena, Instituto de Investigaciones Agropecuarias (INIA). 2007, 68 p. Boletín INIA - no 165.

Brixner, G. F.; Martins, C. R.; Amaral, U. do; Köpp, L. M.; de Oliveira, D. B. Caracterização fenológica e exigência térmica de videiras Vitis vinifera, cultivadas no município de Uruguaiana, na Região da Fronteira Oeste - RS. Revista da FZVA, v.17, p.221-233, 2010.

Brunetto, G.; Ceretta, C. A.; Kaminski, J.; Melo, G. W. B. de; Lourenzi, C. R.; Furlanetto, V.; Moraes, A. Aplicação de nitrogênio em videiras na Campanha Gaúcha: Produtividade e características químicas do mosto da uva. Ciência Rural, v.37, p.389-393, 2007.

Buriol, G. A.; Heldwein, A. B.; Estefanel, V.; Matzenauer, R.; Marcon, I. A. Condições térmicas para o cultivo do pepineiro na região do Baixo Vale do Taquari - RS. Pesquisa Agropecuária Gaúcha, v.6, p.205-213, 2000.

Caramori, P. H.; Caviglione, J. H.; Herter, F. G.; Hauagge, R.; Gonçalves, S. L.; Citadin, I.; Silva, W. da. Zoneamento agroclimático para o pessegueiro e a nectarineira no estado do Paraná. Revista Brasileira de Fruticultura, v.30, p.10401044, 2008.

Chavarria, G.; dos Santos, H. P.; Mandelli, F.; Marodin, G. A. B.; Bergamaschi, H.; Cardoso, L. S. Caracterização fenológica e requerimento térmico da cultivar Moscato Giallo sob cobertura plástica. Revista Brasileira de Fruticultura, v.31, p.119-126, 2009.

Costa, V. B. Efeito das condições climáticas na fenologia da videira européia em Santana do Livramento, Rio Grande do Sul. Pelotas: UFPel, 2011. 89p. Tese Doutorado

Grodzki, L.; Caramori, P. H.; Bootsma, A.; Oliveira, D. de; Gomes, J. Risco de ocorrência de geada no estado do Paraná. Revista Brasileira de Agrometeorologia, v.4, p.93-99, 1996.

Leão, P. C. de S.; Silva, E. E. G. Caracterização fenológica e requerimentos térmicos de variedades de uvas sem sementes no vale do São Francisco. Revista Brasileira de Fruticultura, v.25, p.379-382, 2003.

Maluf, J. R. T.; Matzenauer, R.; Steinmetz, S.; Maluf, D. E. Zoneamento agroclimático da macieira no estado do Rio Grande do Sul. Porto Alegre: FEPAGRO, 2011. Boletim, n.19

R. Bras. Eng. Agríc. Ambiental, v.18, n.2, p.217-224, 2014. 
Maluf, J. R. T.; Westphalen, S. L. Macrozoneamento agroecológico e econômico do estado do Rio Grande do Sul. Porto Alegre: Secretaria da Agricultura e Abastecimento, 1994, v.2, 307p.

Mandelli, F.; Berlato, M. A.; Tonietto, T.; Bergamaschi, Fenologia das videiras na Serra Gaúcha. Pesquisa Agropecuária Gaúcha, v.9, p.129-144, 2003.

Mandelli, F.; Miele, A.; Tonietto, J. Uva em clima temperado. In: Monteiro, J. E. B. A. (Org.). Agrometeorologia dos cultivos: o fator meteorológico na produção agrícola. Brasília: Inmet, 2009. Cap.31, p. 504-515.

Oliveira, H. T. de; Berlato, M. A.; Fontana, D. C. Probabilidade de ocorrência de geada no estado do Rio Grande do Sul. In: Congresso Brasileiro de Agrometeorologia: Agrometeorologia, Monitoramento Ambiental e Agricultura Sustentável, 10. 1997, Piracicaba. Anais... Sociedade Brasileira de Agrometeorologia, 1997. p.77-79.

Pedro Júnior, M. J.; Sentelhas, P. C.; Martins, F. P. Previsão agrometeorologica da data de colheita para a videira 'Niagara Rosada'. Bragantia, v.52, p.113-119, 1994a.

Pedro Júnior, M. J.; Sentelhas, P. C.; Pommer, C. V.; Martins, F. P. Determinação da temperatura-base, graus-dia e índice biometeorológico para a videira 'Niagara Rosada'. Revista Brasileira de Agrometeorologia, v.2, p.51-56, 1994b.

Radünz, A. L. Schöffel, E. D.; Brixner, G. F.; Hallal, M. O. Efeitos da época da poda sobre a duração do ciclo e a produção de videiras 'Bordo' e 'BRS Violeta'. Revista Científica Rural, v.14, p.213-224, 2012.

Regina, M. de A. Viticultura. Revista Brasileira Fruticultura. v.28, p.160-160, 2006.
Rizzon, L. A.; Miele, A. Avaliação da cv. Cabernet Sauvignon para elaboração de vinho tinto. Ciência Tecnologia Alimentos, v.22, p.192-198, 2002.

Schiedeck, G.; Miele, A.; Barradas, C. I. N.; Mandelli, F. Fenologia da videira Niágara Rosada cultivada em estufa e a céu aberto. Revista Brasileira de Agrometeorologia, v.5, p.199-206, 1997.

Silva, J. G. da; Sentelhas, P. C. Diferença de temperatura mínima do ar medida no abrigo e na relva e probabilidade de sua ocorrência em eventos de geada no Estado de Santa Catarina. Revista Brasileira de Agrometeorologia, v.9, p.9-15, 2001.

Tecchio, M. A.; Terra, M. M.; Moura, M. F.; Paioli-Pires, E. J. Fenologia e acúmulo de graus-dia da videira 'Niagara Rosada' cultivada ao noroeste do estado de São Paulo. Revista Brasileira de Fruticultura, v.33, p.248-254, 2011.

Teixeira, A. H. de C.; de Souza, R. A.; Ribeiro, P. H. B.; Reis, V. C. da S.; dos Santos, M. das G. L. Aptidão agroclimática da cultura da videira no Estado da Bahia, Brasil. Revista Brasileira de Engenharia Agrícola e Ambiental, v.6, p.107111, 2002.

Tonietto, J.; Mandelli, F.; Weber, E.; Hasenack, H. Viticultural climatic zoning and digital mapping of Rio Grande do Sul Brazil, using indices of the Géoviticulture MCC System. In: Congrès International des Terroirs Viticoles, 2006, Bordeaux e Montpellier. Anais... 2006, CD.

Wrege, M. S.; Caramori, P. H.; Gonçalves, A C. A.; Bertonha, A.; Ferreira, R. C.; Caviglione, J. H.; Faria, R. T. de; Freitas, P. S. L. de; Gonçalves, S. L. Regiões potenciais para cultivo da cana-de-açúcar no Paraná, com base na análise do risco de geadas. Revista Brasileira de Agrometeorologia, v.13, p.113-122, 2005. 